\title{
Review Article \\ Pathogenesis of Renal Failure in Multiple Myeloma: Any Role of Contrast Media?
}

\author{
Michele Mussap ${ }^{1}$ and Giampaolo Merlini ${ }^{2,3}$ \\ ${ }^{1}$ Laboratory Medicine Service, IRCCS AOU San Martino-IST, National Institute for Cancer Research, University-Hospital San Martino, \\ Largo Rosanna Benzi 10, 16132 Genova, Italy \\ ${ }^{2}$ Amyloidosis Research and Treatment Center, Clinical Chemistry Laboratories, Fondazione Istituto di Ricovero e Cura a Carattere \\ Scientifico (IRCCS) Policlinico San Matteo, Viale Golgi 19, 27100 Pavia, Italy \\ ${ }^{3}$ Department of Molecular Medicine, University of Pavia, Italy
}

Correspondence should be addressed to Giampaolo Merlini; gmerlini@unipv.it

Received 1 November 2013; Revised 13 March 2014; Accepted 13 March 2014; Published 30 April 2014

Academic Editor: Michele Andreucci

Copyright (C) 2014 M. Mussap and G. Merlini. This is an open access article distributed under the Creative Commons Attribution License, which permits unrestricted use, distribution, and reproduction in any medium, provided the original work is properly cited.

The spectrum of kidney disease-associated monoclonal immunoglobulin and plasma cell malignancies is remarkably broad and encompasses nearly all nephropathologic entities. Multiple myeloma with kidney impairment at presentation is a medical emergency since the recovery of kidney function is associated with survival benefits. In most cases, kidney impairment may be the first clinical manifestation of malignant plasma cell dyscrasias like multiple myeloma and light chain amyloidosis. Multiple myeloma per se cannot be considered a main risk factor for developing acute kidney injury following intravascular administration of iodinated contrast media. The risk is increased by comorbidities such as chronic kidney disease, diabetes, hypercalcemia, dehydration, and use of nephrotoxic drugs. Before the administration of contrast media, the current recommended laboratory tests for assessing kidney function are serum creatinine measurement and the estimation of glomerular filtration rate by using the CKD-EPI equation. The assessment of Bence Jones proteinuria is unnecessary for evaluating the risk of kidney failure in patients with multiple myeloma, since this test cannot be considered a surrogate biomarker of kidney function.

\section{Introduction}

The kidney is susceptible to insult from a variety of exogenous (e.g., drugs, organic compounds) and endogenous (toxins, catabolites, etc.) substances because of its complex anatomical, physiological, and biochemical features. Factors contributing to the sensitivity of the kidney include its large blood flow (20-25\% of resting cardiac output corresponding to approximately 1.2-1.3 L/min), concentration of filtered solutes during urine production (kidneys reabsorb more than $99 \%$ of the glomerular filtrate), and the presence of a variety of xenobiotic transporters and metabolizing enzymes. The list of the potential nephrotoxic substances includes drugs, environmental agents, heavy metals, radiocontrast agents, and immunoglobulin free light chains (FLCs). Each of them may adversely affect one or more sites along the nephron, leading to a progressive organ damage and impairment named toxic nephropathy [1]. Toxic nephropathy commonly occurs either as a result of decreased renal perfusion or as direct toxic effects on the proximal tubule; both mechanisms originate specific clinical syndromes (e.g., proximal tubule necrosis, hyperkalemia, medullary thick ascending limb injury). Toxic nephropathy originating from renal or systemic diseases (e.g., nephrotic syndrome, multiple myeloma) can be drastically worsened by such additional conditions (e.g., dehydration, hypoxia), as well as by the effects of potentially nephrotoxic substances (e.g., drugs, contrast media) administered in the course of the disease. The latter condition calls for a careful monitoring of patients in order to avoid the risk of a subsequent nephrotoxic insult leading to severe kidney diseases, like acute tubular necrosis (ATN) and, more extensively, acute kidney injury (AKI). 


\section{Kidney Diseases Associated with Plasma Cells Dyscrasias}

Monoclonal gammopathies refer to a spectrum of disorders characterized by the monoclonal proliferation of lymphoplasmacytic cells in the bone marrow and, sometimes, tissue deposition of monoclonal immunoglobulins (Igs) or their components [2]. The most frequent diseases include monoclonal gammopathy of undetermined significance (MGUS), both asymptomatic (smoldering) and symptomatic multiple myeloma (MM), solitary plasmacytoma, and immunoglobulin light chain amyloidosis (AL amyloidosis) [3]. In patients with $\mathrm{MM}$, the incidence of kidney disease throughout the course of the disease ranges between 15 and 40\%, depending on the definition of kidney injury and failure $[4,5]$. Kidney impairment may be the first clinical manifestation of MM and related conditions, such as light chain amyloidosis [6]. Despite a high rate of partial and complete hematological response of $\mathrm{MM}$ to individually tailored therapies in the novel agent era [7], renal recovery is still poor [8]. MM with renal impairment at presentation should be considered a medical emergency since the recovery of renal function is associated with survival benefit $[9,10]$. The spectrum of kidney disease associated with monoclonal gammopathies is remarkably broad and encompasses nearly all nephropathologic entities (Table 1). The three most common forms of monoclonal immunoglobulin-mediated kidney disease are cast nephropathy, monoclonal immunoglobulin deposition disease (MIDD), and AL amyloidosis. A growing number of pathologic renal conditions are being attributed to a clonal plasma cell disorder that is less "myeloma-like" and more "MGUS-like" in terms of its bulk and proliferative rate [11-13]. Uncommon conditions reported in Table 1 have been recently grouped in the new entity of monoclonal gammopathy of renal significance (MGRS) with the intent to discriminate the pathologic nature of these diseases from the truly benign MGUS [14]. The tubulointerstitial injury, cast nephropathy, is the most common cause of severe AKI in patients with MM ( $\cong 90 \%$ of cases). Data both from the US Renal Data System and from the European Renal Association-European Dialysis and Transplant Association Registry reveal that 1.5\% of patients placed on renal replacement therapy have $\mathrm{MM}$; in patients with end stage renal disease (ESRD) due to MM, the mortality rate is $58 \%$ compared with $31 \%$ in all other ESRD patients [15-17]. Despite the high heterogeneity of kidney disease in plasma cell dyscrasias, the risk of progression to severe organ impairment leading to renal replacement therapy is considerable; thus, it is mandatory to identify patients at risk for kidney damage at a very early stage and to institute treatment promptly. In addition, there is a need for accurate monitoring of these patients to avoid or at least to delay the development of ESRD and dialysis.

\section{Pathogenesis of Kidney Failure in Malignant Plasma Cells Dyscrasias}

In MM, the high monoclonal serum FLCs concentration results in a burden of FLCs on the proximal tubule that
TABLE 1: Kidney disease in plasma cell dyscrasias ([14, 18]).

(I) Common

(1) Light-chain cast nephropathy (myeloma kidney)

(2) Immunoglobulin-related amyloidosis (AL, AHL, and AH)

(3) Monoclonal immunoglobulin deposition disease (LCDD, LHCDD, and HCDD)

(4) Acute tubular necrosis

(A) Drugs (nonsteroidal anti-inflammatory drugs and bisphosphonates)

(B) Intravascular iodinated contrast

(5) Type I and type II cryoglobulinemic glomerulonephritis

(II) Uncommon

(1) Light chain proximal tubulopathy (with or without Fanconi syndrome)

(2) Crystal-storing histiocytosis

(3) Nonamyloid monoclonal fibrillary glomerulonephritis

(4) Immunotactoid glomerulonephritis/glomerulonephritis with organized microtubular monoclonal immunoglobulin deposits (GOMMID)

(5) C3 glomerulonephritis associated with monoclonal gammopathy

(6) Proliferative glomerulonephritis with monoclonal Ig deposits

(7) Hyperviscosity syndrome

(A) Waldenström's macroglobulinemia

(B) $\operatorname{IgM}, \operatorname{IgA}$, and rarely $\operatorname{IgG}$ myeloma

(8) Plasma cell infiltration

(9) Pyelonephritis

(10) Uric acid nephropathy

overwhelms the capacity of both the megalin and cubilin receptors to reabsorb the FLCs. As a consequence, large amounts of FLCs reach the distal tubule lumen where they interact specifically with Tamm-Horsfall proteins (THPs; also known as uromodulin), generating myeloma casts. Cast formation in the distal tubule can block glomerular flow and cause proximal tubular atrophy [19], also contributing to interstitial fibrosis [20]. Simultaneously, the massive reabsorption of monoclonal FLCs within the proximal tubules induces proximal tubule cells apoptosis and DNA degradation, resulting in critical morphologic changes, such as epithelial-to-mesenchymal transition (EMT) or necrosis [21]. In addition, FLCs activate a sequence of inflammatory cascade through nuclear transcription factors, nuclear factor kappa B (NFK B), and AP-1 (c-fos and c-jun) complexes. These transcription factors induce in turn the synthesis of the proinflammatory cytokines interleukin-6 (IL-6), macrophage chemoattractant protein-1 (MCP-1), and tumor necrosis factor $\alpha(\mathrm{TNF} \alpha)$ and activate signaling pathways, such as mitogen-activated protein kinases (MAPK), extracellular signal regulated kinase (ERK1/2), Jun kinase (JNK), and p38 MAPK [22]. As a result, the peculiar histologic lesion of the myeloma cast nephropathy consists of a chronic tubulointerstitial nephropathy with marked tubular atrophy, 
laminated intratubular casts, and extensive interstitial fibrosis. Ultimately, renal fibrogenesis is a major mechanism of kidney impairment in multiple myeloma [23].

\section{Mechanisms of Free Light Chains Nephrotoxicity}

The risk of AKI in patients with MM increases with the concentration of FLCs in urine; however, not all monoclonal FLCs are nephrotoxic. Nephrotoxicity appears to be an intrinsic property of some FLCs, as indicated by the recurrence of similar renal lesions after kidney transplantation [24, 25]. From a clinical point of view, this is confirmed by the fact that a number of patients with considerable FLCs proteinuria never develop kidney disease [26]. As FLCs are composed of different amino acids, they have different isoelectric points (pIs) in solution due to the differences in electrical charge of individual amino acids. As the $\mathrm{pH}$ of the solution reaches that of the protein $\mathrm{pI}$, the net protein electrical charge approaches zero and as such protein precipitation becomes more likely. In kidney disease induced by monoclonal FLCs, the pattern of kidney injury depends on both structural peculiarities of monoclonal FLCs, particularly of the variable $(\mathrm{V})$ domain, and environmental factors, such as $\mathrm{pH}$, urea concentration, or local tissue proteolysis. Moreover, intrinsic host factors play a pivotal role in determining both the type and severity of any renal response to a given FLC. The variable (V) region of the immunoglobulin light chains comprises four key sections that come together to form a hydrophobic core and to allow for the variety of antigen binding, containing three highly variable segments, termed complementary determining regions (CDR), which are attached to joining sections $(J)$. As such $\kappa$ light chains comprise $40 \mathrm{~V} \kappa$ and $5 \mathrm{~J} \kappa$ sections, whereas $\lambda$ light chains have $30 \mathrm{~V} \lambda$ and $8 \mathrm{~J} \lambda$. Although designed to bind antigens, the presence of tyrosine and tryptophan allows additional binding possibilities to lipid-rich cell membranes by binding to sphingomyelin [27, 28]. The CDR 3 domain in the $\mathrm{V}$ region of both $\kappa$ and $\lambda$ FLCs interacts with THP; the binding affinities of FLCs for THP are related to the amino acid composition of the CDR3 domain [29]. On the other hand, THP has a binding domain for FLCs consisting of nine amino acids [30]. In Fanconi syndrome, monoclonal FLCs are nearly always members of the $V \kappa 1$ subfamily and are derived from only two germ line genes, IGKV1-39 and IGKV1-33 [31]. The replacement of polar residues by nonpolar or hydrophobic residues in the complementarity determining regions of these monoclonal FLCs can induce resistance of the $\mathrm{V} \kappa$ domain to proteolysis, resulting in light chain crystallization [32]. In AL amyloidosis and LCDD, the pathogenic role of $\mathrm{V}$ regions is suggested by overrepresentation of the $\mathrm{V} \lambda 6$ and $V \kappa 4$ subgroups, respectively, $\mathrm{N}$-glycosylation of the $\mathrm{V}$ region, and substitutions of key amino acids induced by somatic mutations that might account for the propensity of certain FLCs to aggregate and influence tropism of deposition [33]. In AL amyloidosis, a role for $\mathrm{V}$ sequences is also suggested by the high potential of $\mathrm{V}$ domain dimerization in vitro and in vivo [34]. Unfortunately, there are currently no clinically relevant tools for identifying the potential nephrotoxicity of a specific monoclonal FLC.
TABLE 2: Main risk factors for acute kidney injury (AKI) or acute tubular necrosis (ATN) in patients with malignant plasma cell dyscrasias.

(1) Comorbidities: chronic kidney disease, diabetes, aging, hypertension, and cardiovascular disease

(2) Volume depletion (e.g., dehydration, etc.)

(3) Hypercalcemia

(4) Hyperuricemia

(5) Repeated iodinated contrast media administration

(6) Nonsteroid anti-inflammatory drugs

(7) Diuretics

(8) Aminoglycosides

(9) Hyperviscosity syndrome

\section{Risk Factors and Correlated Mechanisms Precipitating Kidney Failure}

Patients with MM are at an increased risk of kidney disease not only from the kidney injuries and damages due to the primary disease, but also from a number of additional concomitant factors that can substantially contribute to worsen kidney function, leading to AKI, ESRD, and ultimately to renal replacement therapy. A list of these risk factors is reported in Table 2. For example, ATN can be precipitated by dehydration in the presence of FLCs $\kappa$ or $\lambda$ that may deposit in the kidney, as described above. The use of loop diuretics may also contribute to cast formation, leading in turn to serum creatinine levels increase. Loop diuretics are an established risk factor for myeloma kidney. In 1990, it was demonstrated that increasing the concentration of sodium chloride facilitated coprecipitation of Bence Jones proteins with human Tamm-Horsfall glycoprotein in vitro [35]. Furosemide promotes intranephronal obstruction by increasing sodium chloride concentration in the distal nephron, the site of cast formation. In a study published in 1992 it was demonstrated that furosemide accelerated in a concentration-dependent manner cast formation and subsequent obstruction of nephrons perfused in vivo with cast-forming Bence Jones protein [36]. Vasoconstriction, as a result of hypercalcemia, and decreased blood flow from the kidneys, as a result of the use of nonsteroidal antiinflammatory drugs (NSAIDs) or aminoglycosides, may also damage the kidneys [37]. Hypercalcemia is the second most common cause of kidney failure in MM [38]. Elevated calcium concentration in renal tubules causes intratubular calcium deposition and vasoconstriction in renal vasculature. The decrease in GFR induces cast precipitation. Hypercalcemia may also lead to nephrogenic diabetes insipidus, which is characterized by antidiuretic hormone (ADH) resistance. With the impairment of renal concentrating ability, polyuria and polydipsia may also develop. Unfortunately, increased diuresis results in hypovolemia and aggravates the prerenal component of renal failure. Hypovolemia induces cast precipitation by increasing FLC concentration in tubule lumen and lower urine flow contributes to intratubular obstruction. NSAIDs are used extensively in patients with multiple 
myeloma to reduce bone pain. They basically block the production of prostaglandins (PGs), which act as vasodilator hormone, via inhibition of cyclooxygenase enzyme activities (COX-1 and COX-2), leading to renal vasoconstriction and to a reduction in the renal blood flow and GFR. PGs blockage also leads to salt and water retention by the inhibition of chloride reabsorption and $\mathrm{ADH}$. Medullary hypoxia is a major consequence of NSAIDs use, as PGE2 is a major factor in matching medullary oxygen supply and demand. In that respect, papillary necrosis is considered to be caused by critical medullary hypoxia determined by NSAIDs.

\section{Contrast Media-Induced Nephropathy and Plasma Cell Dyscrasias}

In most cases, the onset of contrast induced-acute kidney injury (CI-AKI) consists of a transient nonoliguric form of AKI with serum creatinine levels peaking at $48-72 \mathrm{~h}$ after administration of the medium and returning to baseline values within the subsequent ten days [39]. While the threshold level of kidney injury used to define CI-AKI varies across studies, the most commonly employed definition is an increase in the serum creatinine concentration of at least $0.5 \mathrm{mg} / \mathrm{dL}$ and/or 25\% within 3-4 days of contrast exposure [40]. Appreciable nephropathy is unlikely to develop if the serum creatinine level does not increase by more than $0.5 \mathrm{mg} / \mathrm{dL}$ within 24 hours [41]. Some patients develop a chronic reduction in kidney function or a permanent need for renal replacement therapy. A prospective study showed that the percentage change of serum creatinine $12 \mathrm{~h}$ after contrast versus the basal value was the best predictor of CI-AKI $(P<$ 0.001 ) [42]. A 5\% increase of serum creatinine level yielded $75 \%$ sensitivity and $72 \%$ specificity, with an area under the curve (AUC) of 0.80 and an odd ratio (OR) of 7.37 for early detection. Furthermore, this 12-hour basal value strongly correlated with the development of renal impairment at 30 days $(P=0.002$; sensitivity $87 \%$, specificity $70 \%$; AUC 0.85 ; OR 13.29). In a study using cystatin $C$ as an early marker for AKI, a cutoff cystatin C increase concentration of $\geq 10 \%$ at 24 hours after contrast media exposure was detected in $21.2 \%$ of patients and was the best cutoff value for the early identification of patients at risk for CI-AKI with a negative predictive value of $100 \%$ and a positive predictive value of $39 \%$ [43]. As in other cases of AKI, it appears that, in patients with CKD, cystatin $\mathrm{C}$ may be a useful marker for the early diagnosis of CI-AKI. Kidney injury resulting from iodinated contrast is potentially preventable [44]. Past efforts to find effective preventive strategies for CI-AKI have focused on four principal approaches: (a) use of less nephrotoxic contrast agents; (b) provision of preemptive renal replacement therapy to remove contrast from the circulation prior to its filtration at the glomerulus; (c) expansion of the intravascular space and enhanced diuresis with intravascular fluids; and (d) utilization of pharmacologic agents to counteract the nephrotoxic effects of contrast media [45].

For a long time, MM was considered a significant risk factor for developing contrast nephropathy [46]. This assumption was not lying on robust evidence-based data but only on early published studies that associated intravascular administration of contrast agent and kidney failure in patients with $\mathrm{MM}$ when using ionic agents [47, 48]. Those studies supported the idea that in patients with MM the intravascular administration of iodinated contrast media leads to the sum of two risk factors: the potential nephrotoxic action of contrast media added to the developing/developed kidney disease in malignant plasma cell dyscrasias [49]. As a consequence, radiologists appear to be very conservative in using iodinated intravascular contrast in patients with MM, even though the relative low incidence of CI-AKI in multiple myeloma patients was early reported to be approximately $0.6-$ $1.25 \%$ compared to a risk of $0.15 \%$ in the general public [50] and more recently $5 \%$ within 48 hours and $15 \%$ within 7 days [51]. Several institutional practice guidelines and epidemiological studies [52] suggest that the presumed risk of CI-AKI can be stratified by using three serum creatinine levels: low risk, serum creatinine less than $1.5 \mathrm{mg} / \mathrm{dL}(<132.6 \mu \mathrm{mol} / \mathrm{L})$, medium risk $1.5-2.0 \mathrm{mg} / \mathrm{dL}(132.6-176.8 \mu \mathrm{mol} / \mathrm{L})$, and high risk more than $2.0 \mathrm{mg} / \mathrm{dL}(>176.8 \mu \mathrm{mol} / \mathrm{L})$. Data from a survey performed in 2006 showed that $36 \%$ of USA radiologists never administer intravascular contrast agent to patients with MM and $47 \%$ do it sometimes [53]; in addition, 56\% of nonradiology physicians consider MM with normal renal function to place the patient at increased risk of adverse events associated with iodinated contrast agent administration [54]. Dehydration, infection, hypercalcemia, and Bence Jones proteinuria, which are conditions very often associated with MM, should be considered the primary risk factors for the development of CI-AKI in myeloma [55]. Therefore, there is a need to reconsider the role of intravascular contrast media as risk factor in patients with malignant plasma cell dyscrasia. Several guidelines, published by groups of expert radiologists, nephrologists, and cardiologists, recommend recognizing patients at risk of developing CI-AKI before injection of intravascular contrast media [56-58]. Main risk factors have been reported to be $\mathrm{CKD}$, diabetes, heart failure, low body mass index, hypotension, sepsis, and aging. However, results obtained in a recent meta-analysis lead to the conclusion that out of all risk factors mentioned in published guidelines only a few are significantly associated with contrastinduced nephropathy after intravenous iodinated contrast medium administration [59], namely, CKD, diabetes, aging above 65 years, and use of NSAIDs. Other risk factors such as hypertension, congestive heart failure, contrast volume, hydration status, and anemia do not appear to have a significant association with the incidence of contrast-induced nephropathy [59]. However, the role of anemia as a risk factor for developing CI-AKI should be reevaluated, especially in patients with multiple myeloma. A couple of very recent studies investigated the incidence of CI-AKI in anemic patients, suggesting that low hemoglobin and low hematocrit values may be considered independent predictors of CIAKI $[60,61]$. Since anemia is highly prevalent in multiple myeloma, being reported in about $70 \%$ of patients with newly diagnosed multiple myeloma $[62,63]$, it may represent an important risk factor. It is strongly recommended that risk assessment and prophylactic strategies should be based on eGFR rather than the absolute level of serum creatinine [64]. 


\section{Diagnosis of Kidney Failure and Injury in Multiple Myeloma}

The standard assessment of renal function in patients with MM includes serum creatinine and creatinine clearance, although both measurements probably underestimate the prevalence of renal dysfunction, because of the additional tubular secretion of creatinine and its dependence on extrarenal factors. In particular, serum creatinine is a retrospective, insensitive, and even deceptive measure of kidney injury. Retrospective because its concentration may result in a very delayed signal even after considerable kidney injury [65]. Insensitive because as much as a $50 \%$ loss of renal function may be required to elevate serum creatinine enough that it comes to medical attention, whereas levels that fall short of this threshold are usually dismissed, despite their known association with excess mortality and prolonged hospitalization. As serum creatinine is affected by tubular secretion and systemic production, changes in its concentration are not specific to tubular injury. Deceptive because serum creatinine level often reflects transient physiologic adaptations to volume changes or the presence of CKD rather than the development of AKI. The degree of kidney impairment in MM should be assessed following the Kidney Disease Improving Global Outcomes (KDIGO) classification [66]. In MM patients with stabilized serum creatinine, the International Myeloma Working Group (IMWG) recommended the modification of diet in renal disease (MDRD) equation for estimating GFR (eGFR) [4]. However, more recent studies are supporting the use of the chronic kidney disease epidemiology collaboration cystatin C-based equations (both CKD-EPI-sCR-CysC and CKD-EPI-CysC) [67]. CKD-EPI equations based on cystatin C detect more MM patients with stages 3-5 kidney failure than equations based only on serum creatinine, namely, the MDRD and the original CKD-EPI equations. Interestingly, CKD-EPI-CysC equation predicts overall survival. It is not surprising that the diagnosis of a paraproteinemic renal lesion is hampered by the general lack of sensitivity and specificity of currently available noninvasive tests. The gold standard for diagnosis remains renal biopsy with demonstrated evidence of deposition of monoclonal proteins in the area of injury [68]. The measurement of serum concentration of the clonal FLCs and that of urine level of albumin may be considered the landmarks for screening algorithms in patients with cast nephropathy [69]. It has been proposed that when the concentration of serum FLCs exceeds $500 \mathrm{mg} / \mathrm{L}$ and proteinuria is mainly composed of immunoglobulin light chains, it is extremely likely that the onset of AKI is due to myeloma kidney; in those cases, renal biopsy is usually unnecessary. On the other hand, significant amount of albumin in urine is frequently associated with amyloidosis or MIDD. Amyloid deposits may be confirmed on biopsy of subcutaneous fat or of one of the minor salivary glands. If both are negative, then a kidney biopsy is required to search for amyloid, MIDD, or an unrelated glomerulopathy such as diabetic nephropathy. Patients with CKD and a monoclonal protein need special care, being at increased risk of AKI; renal biopsy is necessary to determine the cause of kidney damage.

\section{Novel Biomarkers of Toxic Nephropathy and Contrast-Induced Nephrotoxicity}

Identification of novel toxic nephropathy and AKI biomarkers has been designated as a top priority by the American Society of Nephrology. The concept of developing a new toolbox for earlier diagnosis of disease states is also prominently featured in the National Institute of Health (NIH) road map for biomedical research. In 2007, the Acute Kidney Injury Network (AKIN), a collaborative group of investigators from all major critical care and nephrology societies, proposed a staging system based on serum creatinine and urine output and consisting of 3 categories (mild, moderate, and severe) in a way similar to those (risk, injury, and failure) used by the RIFLE staging system. AKIN criteria have been included and recommended in the recent AKI guideline published by the Kidney Disease: Improving Global Outcomes (KDIGO) [70]. Despite these working classification systems, the diagnosis of AKI is problematic, as current diagnoses rely on two functional abnormalities: functional changes in serum creatinine and oliguria. Both of these are late consequences of injury and not markers of the injury itself. Most importantly, the measurement of serum creatinine does not identify the cell type that is acutely injured, even though this localization determines the natural history of the disease and its response to therapy. Neutrophil gelatinase-associated lipocalin (NGAL) has emerged as the most promising marker of AKI in a number of clearly defined clinical contexts [71]. NGAL, also known as $l c n 2$, is one of the most upregulated transcripts in the early postischemic mouse kidney and this finding has been confirmed in several other transcriptome profiling studies. Downstream proteomic studies have also revealed NGAL to be one of the earliest and most robustly induced proteins in the kidney after ischemic or nephrotoxic AKI in animal models, and NGAL protein is easily detected in the blood and urine soon after AKI [72]. These findings have spawned a number of translational proteomic studies to evaluate NGAL as a novel biomarker in human AKI. A second very promising biomarker of kidney damage is kidney injury molecule 1 (KIM-1), a type-1 transmembrane glycosylated protein with IgG-like domains in the ectodomain of the protein. Downstream proteomic studies have also shown KIM-1 to be one of the most highly induced proteins in the kidney after AKI in animal models, and a proteolytically processed domain of KIM-1 is easily detected in the urine soon after AKI [73]. KIM-1 represents a promising candidate for inclusion in the urinary "AKI panel" [74, 75]. An advantage of KIM-1 over NGAL is that it appears to be more specific to ischemic or nephrotoxic AKI and not significantly affected by prerenal azotemia, urinary tract infections, or chronic kidney disease. It is likely that NGAL and KIM-1 will emerge as tandem biomarkers of AKI, with NGAL being most sensitive at the earliest time points and KIM-1 adding significant specificity at slightly later time points. In a small human cross-sectional study, KIM-1 was found to be markedly induced in proximal tubules in kidney biopsies from patients with established AKI (primarily ischemic). However, patients with AKI induced by contrast media did not have significant increase in urinary KIM-1 concentration [76]. Additional promising biomarkers 
for the early diagnosis and management of CI-AKI may be the cysteine rich protein 61 (CYR61), induced in the kidney within one hour after ischemic/toxic injury and detectable in the urine 3-6 hours later [77], Krüppel-like factor 6 (Zf9), a Krüppel-like transcription factor involved in the regulation of a number of downstream targets [78], thrombospondin 1 (TSP-1), a p53-dependent proapoptotic and antiangiogenic molecule [79], and IL-18, a proinflammatory cytokine that is known to be induced and cleaved in the proximal tubule and subsequently easily detected in the urine following ischemic AKI in animal models [80]. New encouraging perspectives for improving the current state in clinical diagnosis and in the identification of nephrotoxic injuries are emerging from metabolomics studies [81]. Metabolomics aims to identify unique fingerprints of specific injuries and functions, for example, diseases or effects of exposure to toxic compounds [82]. Predictive models based upon proton nuclear magnetic resonance spectroscopy $\left({ }^{1} \mathrm{H}\right.$ NMR) of rodent urine and serum demonstrated that liver and kidney toxicity can be predicted with sensitivities of 41 and $67 \%$ and specificities of $100 \%$ and $77 \%$, respectively [83].

\section{Clinical Approach to a Patient with Plasma Cell Dyscrasia about to Undergo Radiocontrast Studies}

Although plasma cell dyscrasia would not be "per se" risk factors for the occurrence of CI-AKI, when comorbidities are present, patients require to be carefully monitored after contrast media injection. Kidney impairment, a major risk factor for developing CI-AKI, is one of the more frequent clinical features of symptomatic multiple myeloma. Other coexisting morbidities include anemia, hypercalcemia, and potentially nephrotoxic drugs like NSAIDs, chemotherapeutic agents, and antibiotics. Often, multiple factors may coexist, like kidney impairment with anemia, infections with nephrotoxic drugs, diabetes with aging, and increasing the risk of CI-AKI [84]. The management of patients with plasma cell dyscrasia requiring the intravascular administration of iodinate contrast media should include (a) assessment of the risk before administration; (b) pharmacological and nonpharmacological preventive measures for reducing such risk factors; (c) monitoring after administration; (d) early accurate diagnosis of CI-AKI.

According to the 2012 KDIGO AKI guideline [70], before the intravascular administration of iodinate contrast media, it is mandatory to assess the population at risk by measuring serum creatinine together with the estimation of GFR by the CKD-EPI equation. It has been reported that the incidence of CI-AKI became significant from a baseline serum creatinine concentration of $>1.8 \mathrm{mg} / \mathrm{dL}(>159 \mu \mathrm{mol} / \mathrm{L})$ [85]. However, the CI-AKI Consensus Working Panel agreed that the risk of CI-AKI becomes clinically important when the baseline serum creatinine concentration is $\geq 1.3 \mathrm{mg} / \mathrm{dL}(\geq 115 \mu \mathrm{mol} / \mathrm{L})$ in men and $\geq 1.0 \mathrm{mg} / \mathrm{dL}(\geq 88.4 \mu \mathrm{mol} / \mathrm{L})$ in women [86]. The guideline also recommends that precautions to reduce the risk should be implemented in patients with a baseline eGFR $<45 \mathrm{~mL} / \mathrm{min}$ per $1.73 \mathrm{~m}^{2}$ [70]. When a recent serum creatinine value is not available, a simple questionnaire (e.g., the Choyke questionnaire) [87] or a dipstick testing for urine protein may be useful for identifying preexisting kidney disease. However, dipstick testing is reliable for the identification of albuminuria but it is not able to identify Bence Jones proteinuria. Since serum $\beta_{2}$-microglobulin increases with both higher tumor burden and diminished renal function, it was suggested that evaluation of $\beta_{2}$-microglobulin levels may be useful before intravenous injection of iodinate contrast agents to patients with multiple myeloma [51]. Serum $\beta_{2}$ microglobulin likely serves as a marker of patients who are at a higher risk of developing CI-AKI. According to previous results published elsewhere, a value of $\beta_{2}$-microglobulin below $2.8 \mathrm{mg} / \mathrm{L}$ may be the most clinically useful marker for patients at low risk of developing CI-AKI, showing a $100 \%$ negative predictive value [51].

According to the 2012 KDIGO AKI guideline, pharmacological and nonpharmacological preventive measures for reducing risk factors for CI-AKI in patients with multiple myeloma include (a) the use of either iso-osmolar or lowosmolar rather than high-osmolar iodinated contrast media (strength of recommendation 1B); (b) extracellular volume expansion at the time of radiocontrast media administration with either isotonic sodium chloride or sodium bicarbonate solutions (strength of recommendation 1A). It is desirable not to use oral fluids alone (strength of recommendation $1 \mathrm{C}$ ), while it is recommended to use oral $N$-acetylcysteine (NAC) together with intravenous isotonic crystalloids (strength of recommendation 2D). A meta-analysis on 41 studies showed that NAC is the most effective agent for preventing CI-AKI in patients with CKD [88]. Following evidence in animal model that aciduria increases the nephrotoxicity of Bence Jones protein independently of urinary flow rate $[88,89]$, alkalinization of the urine through oral bicarbonate has been recommended in patients with multiple myeloma and Bence Jones proteinuria to prevent or treat myeloma cast nephropathy. In a trial performed in myeloma patients presenting with renal failure, patients were randomized to receive either sodium bicarbonate to render their urine neutral or no supplement. Patients randomized to receive alkali fared marginally better than the others, but the difference was not significant [90]. Urine alkalinization is now recommended by consensus documents on the management of renal failure in multiple myeloma patients [4]. On the basis of recent evidence, it is reasonable to correct marked anemia in patients with mildto-moderate kidney impairment before contrast studies. The combination of kidney disease with anemia significantly increases the risk of CI-AKI with reported incidences of $6.3-7.8 \%$ in anemic patients compared with $2.2-2.8 \%$ in nonanemic patients $[60,61]$. When serum FLCs level exceeds $500 \mathrm{mg} / \mathrm{L}$ in patients with multiple myeloma and other lymphoproliferative disorders, it is reasonable to achieve a rapid reduction in serum FLCs levels before administrating the contrast medium [25]. This emergency treatment is based on high-dose dexamethasone. Bisphosphonates are effective for controlling malignancy-related hypercalcemia; nevertheless, they can further impair renal function and cause symptomatic hypocalcemia in patients with acute renal failure and their use is discouraged in such patients [91]. Although 
the reduction of hypercalcemia has been considered optional in the 2012 KDIGO AKI guideline, we suggest correcting high concentrations of serum calcium before contrast media studies, being a risk factor for acute kidney impairment. Finally, as a preventive measurement in multiple myeloma patients at high risk for CI-AKI, it is desirable to discontinue the therapeutic administration of NSAIDs, aminoglycosides, high dose diuretics, antineoplastic drugs, and metformin 48 hours before contrast media administration.

Multiple myeloma inpatients monitoring consists of measurement of serum creatinine together with the calculation of eGFR at 12 and 48 hours after contrast media injection. If there is evidence of CI-AKI, it is recommended to repeat these tests on days 3-5 after contrast media administration, monitoring also urine output, and it is reasonable to prolong the discontinuation of the therapeutic treatment with NSAIDs, aminoglycosides, high dose diuretics, antineoplastic drugs, and metformin [70].

Finally, diagnosis of CI-AKI can be improved by adding the measurement of serum cystatin $\mathrm{C}$, which allows for an earlier assessment of changes in renal filtration [92]. Urine NGAL may be also useful for assessing the presence of kidney injury and damage, often preexistent in patients treated with nephrotoxic drugs.

\section{Conclusions}

Data from recent meta-analyses have led to the conclusion that the overall incidence of CI-AKI is low, not exceeding $6.4 \%$; moreover, an association between the presence of malignancy with contrast-induced nephropathy was found $[59,93]$. Nevertheless, this could result from comorbidities and the nephrotoxic chemotherapy administered to these patients in combination with intravenous iodinated contrast medium. Further evidence suggests that the incidence of CIAKI might be overestimated. In a retrospective study on more than 50,000 patients divided in two subgroups (intravenous contrast-enhanced and contrast-unenhanced CT scan) it was found that the incidence of CI-AKI was not significantly different from AKI caused by other factors [94], confirming similar results published previously [95]. The hypothesis that CI-AKI might be overestimated was further supported by a meta-analysis including 13 studies and representing 25,950 patients; the incidence of CI-AKI was $6.4 \%$ (ranging from $2.1 \%$ to $19 \%$ ), while the incidence of noncontrast medium AKI was $6.5 \%$ (ranging from $1.3 \%$ to $19.8 \%$ ) [96].

So far, there is limited evidence that myeloma per se may be a risk factor for developing CI-AKI. A careful medical history examination together with serum creatinine and the estimation of GFR by using the equation CKD-EPI-sCr-Cys may be considered the best cost/effective strategy to prevent CI-AKI in patients with multiple myeloma. The use of NGAL and KIM-1 as tandem markers for early detection and later confirmation, respectively, of kidney injury in patients at high risk should be considered. When serum FLCs level exceeds $500 \mathrm{mg} / \mathrm{L}$ in patients with multiple myeloma and other lymphoproliferative disorders, it is reasonable to achieve a rapid reduction in serum FLCs levels before administrating the contrast medium. Additional preventive measures include the use of the lowest volumes required and the removal of concomitantly administered nephrotoxic substances/drugs, especially NSAIDs. We should avoid redundant laboratory tests, like Bence Jones proteinuria, since this test cannot be considered as a surrogate biomarker of kidney function.

\section{Conflict of Interests}

The authors declare that there is no conflict of interests regarding the publication of this paper.

\section{Acknowledgments}

Giampaolo Merlini was supported by a grant from Associazione Italiana per la Ricerca sul Cancro, "Special Program Molecular Clinical Oncology 5 per mille," no. 9965, “Harnessing Tumor Cell/Microenvironment Cross Talk to Treat Mature B cell Tumors," and by a grant from Fondazione Cariplo (Grant no. 2013-0964).

\section{References}

[1] IPCS CEC (International Programme on Chemical Safety, Commission of the European Communities), "Principle and methods for the assessment of nephrotoxicity associated with exposure to chemicals: environmental health criteria 119," Geneva, Switzerland, WHO, 1991.

[2] S. V. Rajkumar, R. A. Kyle, and F. K. Buadi, "Advances in the diagnosis, classification, risk stratification, and management of monoclonal gammopathy of undetermined significance: implications for recategorizing disease entities in the presence of evolving scientific evidence," Mayo Clinic Proceedings, vol. 85, no. 10 , pp. $945-948,2010$.

[3] International Myeloma Working Group, "Criteria for the classification of monoclonal gammopathies, multiple myeloma and related disorders: a report of the International Myeloma Working Group," British Journal of Haematology, vol. 121, no. 5, pp. 749-757, 2003.

[4] M. A. Dimopoulos, E. Terpos, A. Chanan-Khan et al., "Renal impairment in patients with multiple myeloma: a consensus statement on behalf of the International Myeloma Working Group," Journal of Clinical Oncology, vol. 28, no. 33, pp. 49764984, 2010.

[5] E. C. Heher, H. G. Rennke, J. P. Laubach, and P. G. Richardson, "Kidney disease and multiple myeloma," Clinical Journal of the American Society of Nephrology, vol. 8, no. 11, pp. 2007-2017, 2013.

[6] G. Merlini, A. D. Wechalekar, and G. Palladini, "Systemic light chain amyloidosis: an update for treating physicians," Blood, vol. 121, no. 26, pp. 5124-5130, 2013.

[7] C. Rosenbaum, J. Jasielec, J. Laubach, C. P. Prada, P. Richardson, and A. J. Jakubowiak, "Evolving strategies in the initial treatment of multiple myeloma," Seminars in Oncology, vol. 40, no. 5, pp. 592-601, 2013.

[8] S. V. Glavey, M. A. Gertz, A. Dispenzieri et al., "Long-term outcome of patients with multiple myeloma-related advanced renal failure following auto-SCT," Bone Marrow Transplant, vol. 48, pp. 1543-1547, 2013.

[9] E. Kastritis, A. Anagnostopoulos, M. Roussou et al., "Reversibility of renal failure in newly diagnosed multiple myeloma 
patients treated with high dose dexamethasone-containing regimens and the impact of novel agents," Haematologica, vol. 92, no. 4, pp. 546-549, 2007.

[10] R. J. Haynes, S. Read, G. P. Collins, S. C. Darby, and C. G. Winearls, "Presentation and survival of patients with severe acute kidney injury and multiple myeloma: a 20 -year experience from a single centre," Nephrology Dialysis Transplantation, vol. 25, no. 2, pp. 419-426, 2010.

[11] G. Merlini and M. J. Stone, "Dangerous small B-cell clones," Blood, vol. 108, no. 8, pp. 2520-2530, 2006.

[12] S. H. Nasr, A. Satoskar, G. S. Markowitz et al., "Proliferative glomerulonephritis with monoclonal IgG deposits," Journal of the American Society of Nephrology, vol. 20, no. 9, pp. 20552064, 2009.

[13] F. Bridoux, E. Desport, V. Frémeaux-Bacchi et al., "Glomerulonephritis with isolated C3 deposits and monoclonal gammopathy: a fortuitous association?" Clinical Journal of the American Society of Nephrology, vol. 6, no. 9, pp. 2165-2174, 2011.

[14] N. Leung, F. Bridoux, C. A. Hutchison et al., "International kidney and monoclonal gammopathy research group. Monoclonal gammopathy of renal significance: when MGUS is no longer undetermined or insignificant," Blood, vol. 120, pp. 4292-4295, 2012.

[15] K. C. Abbott and L. Y. Agodoa, "Multiple myeloma and light chain-associated nephropathy at end-stage renal disease in the United States: patient characteristics and survival," Clinical Nephrology, vol. 56, no. 3, pp. 207-210, 2001.

[16] D. J. Tsakiris, V. S. Stel, P. Finne et al., "Incidence and outcome of patients starting renal replacement therapy for end-stage renal disease due to multiple myeloma or light-chain deposit disease: an ERA-EDTA Registry study," Nephrology Dialysis Transplantation, vol. 25, no. 4, pp. 1200-1206, 2010.

[17] V. Eleutherakis-Papaiakovou, A. Bamias, D. Gika et al., "Renal failure in multiple myeloma: incidence, correlations, and prognostic significance," Leukemia and Lymphoma, vol. 48, no. 2, pp. 337-341, 2007.

[18] N. Leung and S. V. Rajkumar, "Renal manifestations of plasma cell disorders," American Journal of Kidney Diseases, vol. 50, no. 1, pp. 155-165, 2007.

[19] G. A. Herrera and P. W. Sanders, "Paraproteinemic renal diseases that involve the tubulo-interstitium," Contributions to Nephrology, vol. 153, pp. 105-115, 2007.

[20] M. A. Dimopoulos, E. Kastritis, L. Rosinol, J. Bladé, and H. Ludwig, "Pathogenesis and treatment of renal failure in multiple myeloma," Leukemia, vol. 22, no. 8, pp. 1485-1493, 2008.

[21] M. Li, K. S. Hering-Smith, E. E. Simon, and V. Batuman, "Myeloma light chains induce epithelial-mesenchymal transition in human renal proximal tubule epithelial cells," Nephrology Dialysis Transplantation, vol. 23, no. 3, pp. 860-870, 2008.

[22] S. Sengul, C. Zwizinski, and V. Batuman, "Role of MAPK pathways in light chain-induced cytokine production in human proximal tubule cells," American Journal of Physiology-Renal Physiology, vol. 284, no. 6, pp. F1245-F1254, 2003.

[23] G. A. Herrera, L. Joseph, X. Gu, A. Hough, and B. Barlogie, "Renal pathologic spectrum in an autopsy series of patients with plasma cell dyscrasia," Archives of Pathology and Laboratory Medicine, vol. 128, no. 8, pp. 875-879, 2004.

[24] A. A. Khamlichi, A. Rocca, G. Touchard, P. Aucouturier, J.L. Preud'Homme, and M. Cogné, "Role of light chain variable region in myeloma with light chain deposition disease: evidence from an experimental model," Blood, vol. 86, no. 10, pp. 36553659, 1995.
[25] C. A. Hutchison, V. Batuman, J. Behrens et al., "The pathogenesis and diagnosis of acute kidney injury in multiple myeloma," Nature Reviews Nephrology, vol. 8, no. 1, pp. 43-51, 2011.

[26] R. Woodruff and B. Sweet, "Multiple myeloma with massive Bence Jones proteinuria and preservation of renal function," Australian and New Zealand Journal of Medicine, vol. 7, no. 1, pp. 60-62, 1977.

[27] A. T. Hutchinson, P. A. Ramsland, D. R. Jones et al., "Free Ig light chains interact with sphingomyelin and are found on the surface of myeloma plasma cells in an aggregated form," The Journal of Immunology, vol. 185, no. 7, pp. 4179-4188, 2010.

[28] A. T. Hutchinson, A. Malik, M. B. Berkahn et al., "Formation of assemblies on cell membranes by secreted proteins: molecular studies of free $\lambda$ light chain aggregates found on the surface of myeloma cells," The Biochemical Journal, vol. 454, pp. 479-489, 2013.

[29] W.-Z. Ying and P. W. Sanders, "Mapping the binding domain of immunoglobulin light chains for Tamm-Horsfall protein," The American Journal of Pathology, vol. 158, no. 5, pp. 1859-1866, 2001.

[30] Z.-Q. Huang and P. W. Sanders, "Localization of a single binding site for immunoglobulin light chains on human Tamm-Horsfall glycoprotein," The Journal of Clinical Investigation, vol. 99, no. 4, pp. 732-736, 1997.

[31] A. Rocca, A. A. Khamlichi, G. Touchard et al., "Sequences of $\mathrm{V} \kappa \mathrm{L}$ subgroup light chains in Fanconi’s syndrome: light chain $\mathrm{V}$ region gene usage restriction and peculiarities in myelomaassociated Fanconi's syndrome," The Journal of Immunology, vol. 155, no. 6, pp. 3245-3252, 1995.

[32] T. Messiaen, S. Deret, B. Mougenot et al., "Adult Fanconi syndrome secondary to light chain gammopathy: clinicopathologic heterogeneity and unusual features in 11 patients," Medicine, vol. 79, no. 3, pp. 135-154, 2000.

[33] L. M. Blancas-Mejia, L. A. Tellez, L. del Pozo-Yauner, B. Becerril, J. M. Sanchez-Ruiz, and D. A. Fernandez-Velasco, "Thermodynamic and kinetic characterization of a germ line human $\lambda 6$ light-chain protein: the relation between unfolding and fibrillogenesis," Journal of Molecular Biology, vol. 386, no. 4, pp. 1153-1166, 2009.

[34] B. Kaplan, M. Ramirez-Alvarado, L. Sikkink et al., "Free light chains in plasma of patients with light chain amyloidosis and non-amyloid light chain deposition disease. High proportion and heterogeneity of disulfide-linked monoclonal free light chains as pathogenic features of amyloid disease," British Journal of Haematology, vol. 144, no. 5, pp. 705-715, 2009.

[35] P. W. Sanders, B. B. Booker, J. B. Bishop, and H. C. Cheung, "Mechanisms of intranephronal proteinaceous cast formation by low molecular weight proteins," The Journal of Clinical Investigation, vol. 85, no. 2, pp. 570-576, 1990.

[36] P. W. Sanders and B. B. Booker, "Pathobiology of cast nephropathy from human Bence Jones proteins," The Journal of Clinical Investigation, vol. 89, no. 2, pp. 630-639, 1992.

[37] M. A. Dimopoulos and E. Terpos, "Renal insufficiency and failure," Hematology/The Education Program of the American Society of Hematology, vol. 2010, pp. 431-436, 2010.

[38] J. Bladé and R. L. Renal, "Hematologic and infectious complications in multiple myeloma," Best Practice \& Research Clinical Haematology, vol. 18, no. 4, pp. 635-652, 2005.

[39] R. Solomon, "Contrast-medium-induced acute renal failure," Kidney International, vol. 53, pp. 230-242, 1998. 
[40] R. Mehran and E. Nikolsky, "Contrast-induced nephropathy: definition, epidemiology, and patients at risk," Kidney International, vol. 69, no. 100, pp. S11-S15, 2006.

[41] N. V. Guttterez, A. Diaz, G. C. Timmis et al., "Determinants of serum creatinine trajectory in acute contrast nephropathy," Journal of Interventional Cardiology, vol. 15, no. 5, pp. 349-354, 2002.

[42] F. Ribichini, M. Graziani, G. Gambaro et al., "Early creatinine shifts predict contrast-induced nephropathy and persistent renal damage after angiography," The American Journal of Medicine, vol. 123, no. 8, pp. 755-763, 2010.

[43] C. Briguori, G. Visconti, N. V. Rivera et al., "Cystatin C and contrast-induced acute kidney injury," Circulation, vol. 121, no. 19, pp. 2117-2122, 2010.

[44] B. J. Barrett and P. S. Parfrey, "Preventing nephropathy induced by contrast medium," The New England Journal of Medicine, vol. 354, no. 4, pp. 379-386, 2006.

[45] S. D. Weisbord and P. M. Palevsky, "Strategies for the prevention of contrast-induced acute kidney injury," Current Opinion in Nephrology and Hypertension, vol. 19, no. 6, pp. 539-549, 2010.

[46] D. J. Cohen, W. H. Sherman, E. F. Osserman, and G. B. Appel, "Acute renal failure in patients with multiple myeloma," The American Journal of Medicine, vol. 76, no. 2, pp. 247-256, 1984.

[47] E. D. Bartels, G. C. Brun, A. Gammeltoft, and P. A. Gjørup, "Acute anuria following intravenous pyelography in a patient with myelomatosis," Acta Medica Scandinavica, vol. 150, no. 4, pp. 297-302, 1954.

[48] P. E. Perillie and H. O. Conn, "Acute renal failure after intravenous pyelography in plasma cell myeloma," The Journal of the American Medical Association, vol. 167, no. 18, pp. 2186-2189, 1958.

[49] G. H. Myers Jr. and D. M. Witten, "Acute renal failure after excretory urography in multiple myeloma," The American Journal of Roentgenology, Radium Therapy, and Nuclear Medicine, vol. 113, no. 3, pp. 583-588, 1971.

[50] C. S. McCarthy and J. A. Becker, "Multiple myeloma and contrast media," Radiology, vol. 183, no. 2, pp. 519-521, 1992.

[51] J. K. Pahade, C. A. LeBedis, V. D. Raptopoulos et al., "Incidence of contrast-induced nephropathy in patients with multiple myeloma undergoing contrast-enhanced CT,' American Journal of Roentgenology, vol. 196, no. 5, pp. 1094-1101, 2011.

[52] R. Solomon and W. Dumouchel, "Contrast media and nephropathy: findings from systematic analysis and food and drug administration reports of adverse effects," Investigative Radiology, vol. 41, no. 8, pp. 651-660, 2006.

[53] B. M. Elicker, Y. S. Cypel, and J. C. Weinreb, "IV contrast administration for CT: a survey of practices for the screening prevention of contrast nephropathy," American Journal of Roentgenology, vol. 186, no. 6, pp. 1651-1658, 2006.

[54] E. L. I. Konen, O. Konen, M. Katz, Y. Levy, J. Rozenman, and M. Hertz, "Are referring clinicians aware of patients at risk from intravenous injection of iodinated contrast media?" Clinical Radiology, vol. 57, no. 2, pp. 132-135, 2002.

[55] R. A. DeFronzo, R. L. Humphrey, J. R. Wright, and C. R. Cooke, "Acute renal failure in multiple myeloma," Medicine, vol. 54, no. 3, pp. 209-223, 1975.

[56] L. Lepanto, A. Tang, J. Murphy-Lavallée, and J.-S. Billiard, “The Canadian Association of Radiologists guidelines for the prevention of contrast-induced nephropathy: a critical appraisal," Canadian Association of Radiologists Journal, vol. 62, no. 4, pp. 238-242, 2011.
[57] M. F. Bellin, O. Clement, and G. Heinz-Peer, "Contrast media safety committee of European society of urogenital radiology (ESUR). Contrast induced nephropathy: updated ESUR contrast media safety committee guidelines," European Radiology , vol. 21, pp. 2527-2541, 2011.

[58] American College of Radiology, ACR Manual on Contrast Media, version 7, American College of Radiology, Reston, Va, USA, 2010.

[59] S. I. Moos, D. N. van Vemde, J. Stoker, and S. Bipat, "Contrast induced nephropathy in patients undergoing intravenous (IV) contrast enhanced computed tomography (CECT) and the relationship with risk factors: a meta-analysis," European Journal of Radiology, vol. 82, no. 3, pp. e387-e399, 2013.

[60] W.-H. Li, D.-Y. Li, F. Han, T.-D. Xu, Y.-B. Zhang, and H. Zhu, "Impact of anemia on contrast-induced nephropathy (CIN) in patients undergoing percutaneous coronary interventions," International Urology and Nephrology, vol. 45, no. 4, pp. 10651070, 2013.

[61] R. Murakami, S. Kumita, H. Hayashi et al., "Anemia and the risk of contrast-induced nephropathy in patients with renal insufficiency undergoing contrast-enhanced MDCT," European Journal of Radiology, vol. 82, no. 10, pp. e521-e524, 2013.

[62] G. Birgegård, P. Gascón, and H. Ludwig, "Evaluation of anaemia in patients with multiple myeloma and lymphoma: findings of the European Cancer Anaemia Survey," European Journal of Haematology, vol. 77, no. 5, pp. 378-386, 2006.

[63] R. A. Kyle, M. A. Gertz, T. E. Witzig et al., "Review of 1027 patients with newly diagnosed multiple myeloma," Mayo Clinic Proceedings, vol. 78, no. 1, pp. 21-33, 2003.

[64] The Ad-Hoc Working Group of ERBP, D. Fliser, M. Laville et al., "A European renal best practice (ERBP) position statement on the kidney disease improving global outcomes (KDIGO) clinical practice guidelines on acute kidney injury-part 1: definitions, conservative management and contrast-induced nephropathy," Nephrology Dialysis Transplantation, vol. 27, no. 12, pp. 4263-4272, 2012.

[65] S. S. Waikar and J. V. Bonventre, "Creatinine kinetics and the definition of acute kidney injury," Journal of the American Society of Nephrology, vol. 20, no. 3, pp. 672-679, 2009.

[66] A. S. Levey, K.-U. Eckardt, Y. Tsukamoto et al., "Definition and classification of chronic kidney disease: a position statement from kidney disease: improving global outcomes (KDIGO)," Kidney International, vol. 67, no. 6, pp. 2089-2100, 2005.

[67] E. Terpos, D. Christoulas, E. Kastritis et al., "The chronic kidney disease epidemiology collaboration cystatin C, (CKDEPI-CysC) equation has an independent prognostic value for overall survival in newly diagnosed patients with symptomatic multiple myeloma, is it time to change from MDRD to CKDEPI-CysC equations?" European Journal of Haematology, vol. 91, no. 4, pp. 347-355, 2013.

[68] G. A. Herrera and P. W. Sanders, "Paraproteinemic renal diseases that involve the tubulo-interstitium," in The Kidney in Plasma Cell Dyscrasias, G. A. Herrera, Ed., vol. 153, pp. 105-115, Karger, Basel, Switzerland, 2007.

[69] A. Davenport and G. Merlini, "Myeloma kidney: advances in molecular mechanisms of acute kidney injury open novel therapeutic opportunities," Nephrology Dialysis Transplantation, vol. 27, no. 10, pp. 3713-3718, 2012.

[70] Kidney Disease: Improving Global Outcomes (KDIGO) Acute Kidney Injury Work Group, "KDIGO clinical practice guideline for acute kidney injury," Kidney International Supplements, vol. 2, pp. 1-138, 2012. 
[71] S. Supavekin, W. Zhang, R. Kucherlapati, F. J. Kaskel, L. C. Moore, and P. Devarajan, "Differential gene expression following early renal ischemia/reperfusion," Kidney International, vol. 63, no. 5, pp. 1714-1724, 2003.

[72] K. Mori, H. T. Lee, D. Rapoport et al., "Endocytic delivery of lipocalin-siderophore-iron complex rescues the kidney from ischemia-reperfusion injury," The Journal of Clinical Investigation, vol. 115, no. 3, pp. 610-621, 2005.

[73] J. V. Bonventre, "Kidney injury molecule-1 (KIM-1): a urinary biomarker and much more," Nephrology Dialysis Transplantation, vol. 24, no. 11, pp. 3265-3268, 2009.

[74] J. Fontanilla and W. K. Han, "Kidney injury molecule-1 as an early detection tool for acute kidney injury and other kidney diseases," Expert Opinion on Medical Diagnostics, vol. 5, no. 2, pp. 161-173, 2011.

[75] T. Ichimura, C. C. Hung, S. A. Yang, J. L. Stevens, and J. V. Bonventre, "Kidney injury molecule-1: a tissue and urinary biomarker for nephrotoxicant-induced renal injury," American Journal of Physiology: Renal Physiology, vol. 286, no. 3, pp. F552F563, 2004.

[76] W. K. Han, V. Bailly, R. Abichandani, R. Thadhani, and J. V. Bonventre, "Kidney injury molecule-1 (KIM-1): a novel biomarker for human renal proximal tubule injury," Kidney International, vol. 62, no. 1, pp. 237-244, 2002.

[77] Y. Muramatsu, M. Tsujie, Y. Kohda et al., "Early detection of cysteine rich protein 61 (CYR61, CCN1) in urine following renal ischemic reperfusion injury," Kidney International, vol. 62, no. 5, pp. 1601-1610, 2002.

[78] R. Tarabishi, K. Zahedi, J. Mishra et al., "Induction of Zf9 in the kidney following early ischemia/reperfusion," Kidney International, vol. 68, no. 4, pp. 1511-1519, 2005.

[79] C. V. Thakar, K. Zahedi, M. P. Revelo et al., "Identification of thrombospondin 1 (TSP-1) as a novel mediator of cell injury in kidney ischemia," The Journal of Clinical Investigation, vol. 115, no. 12, pp. 3451-3459, 2005.

[80] C. R. Parikh, A. Jani, V. Y. Melnikov, S. Faubel, and C. L. Edelstein, "Urinary interleukin-18 is a marker of human acute tubular necrosis," American Journal of Kidney Diseases, vol. 43, no. 3, pp. 405-414, 2004.

[81] J. K. Nicholson, J. Connelly, J. C. Lindon, and E. Holmes, "Metabonomics: a platform for studying drug toxicity and gene function," Nature Reviews Drug Discovery, vol. 1, no. 2, pp. 153$161,2002$.

[82] J. D. Stewart and H. M. Bolt, "Metabolomics: biomarkers of disease and drug toxicity," Archives of Toxicology, vol. 85, no. 1 , pp. 3-4, 2011.

[83] R. D. Beger, J. Sun, and L. K. Schnackenberg, "Metabolomics approaches for discovering biomarkers of drug-induced hepatotoxicity and nephrotoxicity," Toxicology and Applied Pharmacology, vol. 243, no. 2, pp. 154-166, 2010.

[84] J. Bladé and L. Rosiñol, "Complications of multiple myeloma," Hematology/Oncology Clinics of North America, vol. 21, no. 6, pp. 1231-1246, 2007.

[85] R. J. Bruce, A. Djamali, K. Shinki, S. J. Michel, J. P. Fine, and M. A. Pozniak, "Background fluctuation of kidney function versus contrast-induced nephrotoxicity," American Journal of Roentgenology, vol. 192, no. 3, pp. 711-718, 2009.

[86] N. Lameire, A. Adam, C. R. Becker et al., "Baseline renal function screening," The American Journal of Cardiology, vol. 98, supplement 1, no. 6, pp. 21-26, 2006.
[87] H. S. Thomsen and S. K. Morcos, "Contrast media and the kidney: European Society of Urogenital Radiology (ESUR) guidelines," British Journal of Radiology, vol. 76, no. 908, pp. 513518, 2003.

[88] M. D. Holland, J. H. Galla, P. W. Sanders, and R. G. Luke, "Effect of urinary $\mathrm{pH}$ and diatrizoate on Bence Jones protein nephrotoxicity in the rat," Kidney International, vol. 27, no. 1, pp. 46-50, 1985.

[89] D. H. Clyne and V. E. Pollak, "Renal handling and pathophysiology of Bence Jones proteins," Contributions to Nephrology, vol. 24, pp. 78-87, 1981.

[90] MRC Working Party on Leukaemia in Adults, "Analysis and management of renal failure in fourth MRC myelomatosis trial," British Medical Journal, vol. 288, no. 6428, pp. 1411-1416, 1984.

[91] M. A. Perazella and G. S. Markowitz, "Bisphosphonate nephrotoxicity," Kidney International, vol. 74, no. 11, pp. 1385-1393, 2008.

[92] F. Ribichini, G. Gambaro, M. S. Graziani et al., "Comparison of serum creatinine and cystatin $\mathrm{C}$ for early diagnosis of contrast-induced nephropathy after coronary angiography and interventions," Clinical Chemistry, vol. 58, no. 2, pp. 458-464, 2012.

[93] J. Kooiman, S. M. Pasha, W. Zondag et al., "Meta-analysis: serum creatinine changes following contrast enhanced CT imaging," European Journal of Radiology, vol. 81, no. 10, pp. 2554-2561, 2012.

[94] R. J. McDonald, J. S. McDonald, J. P. Bida et al., "Intravenous contrast material-induced nephropathy: causal or coincident phenomenon?" Radiology, vol. 267, no. 1, pp. 106-118, 2013.

[95] J. H. Newhouse, D. Kho, Q. A. Rao, and J. Starren, "Frequency of serum creatinine changes in the absence of iodinated contrast material: implications for studies of contrast nephrotoxicity," American Journal of Roentgenology, vol. 191, no. 2, pp. 376-382, 2008.

[96] J. S. McDonald, R. J. McDonald, J. Comin et al., "Frequency of acute kidney injury following intravenous contrast medium administration: a systematic review and meta-analysis," Radiology, vol. 267, no. 1, pp. 119-128, 2013. 


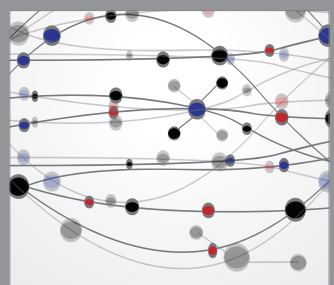

The Scientific World Journal
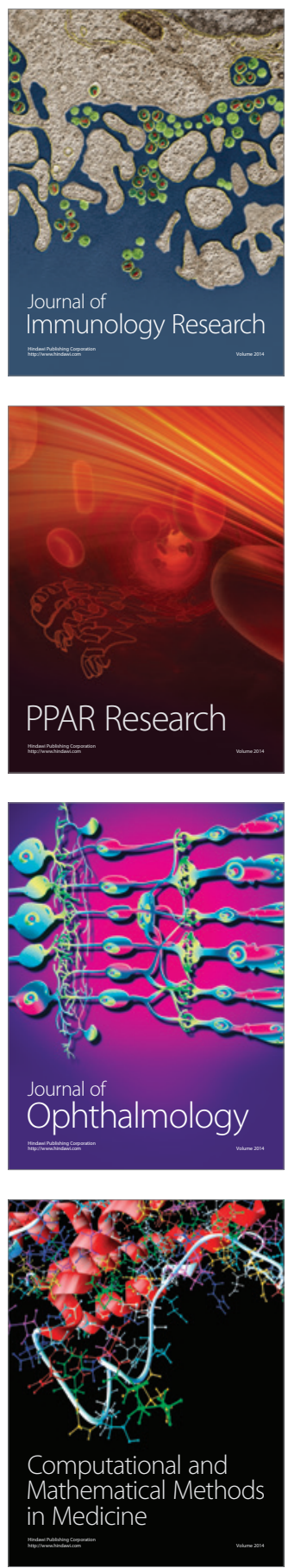

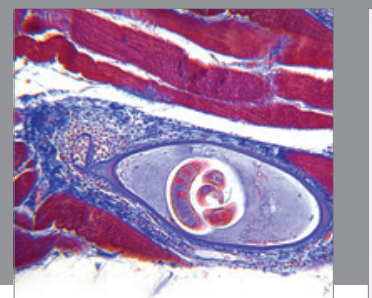

Gastroenterology

Research and Practice
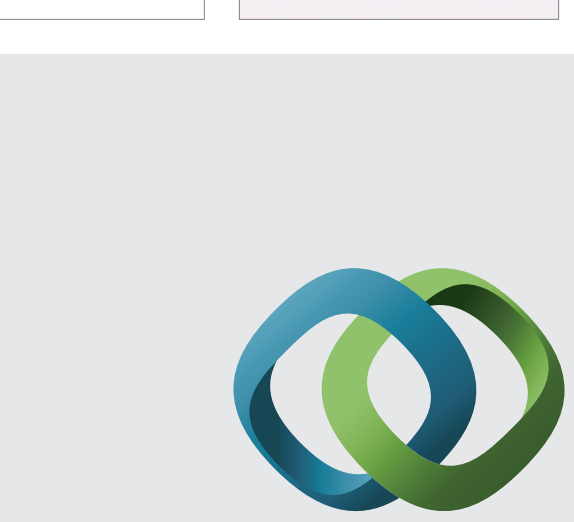

\section{Hindawi}

Submit your manuscripts at

http://www.hindawi.com
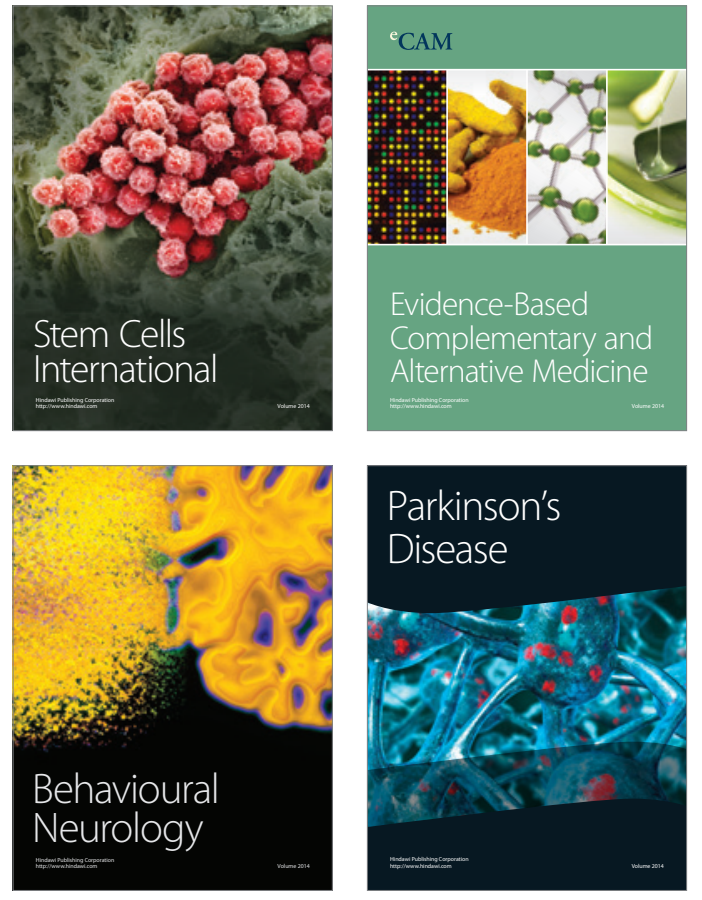
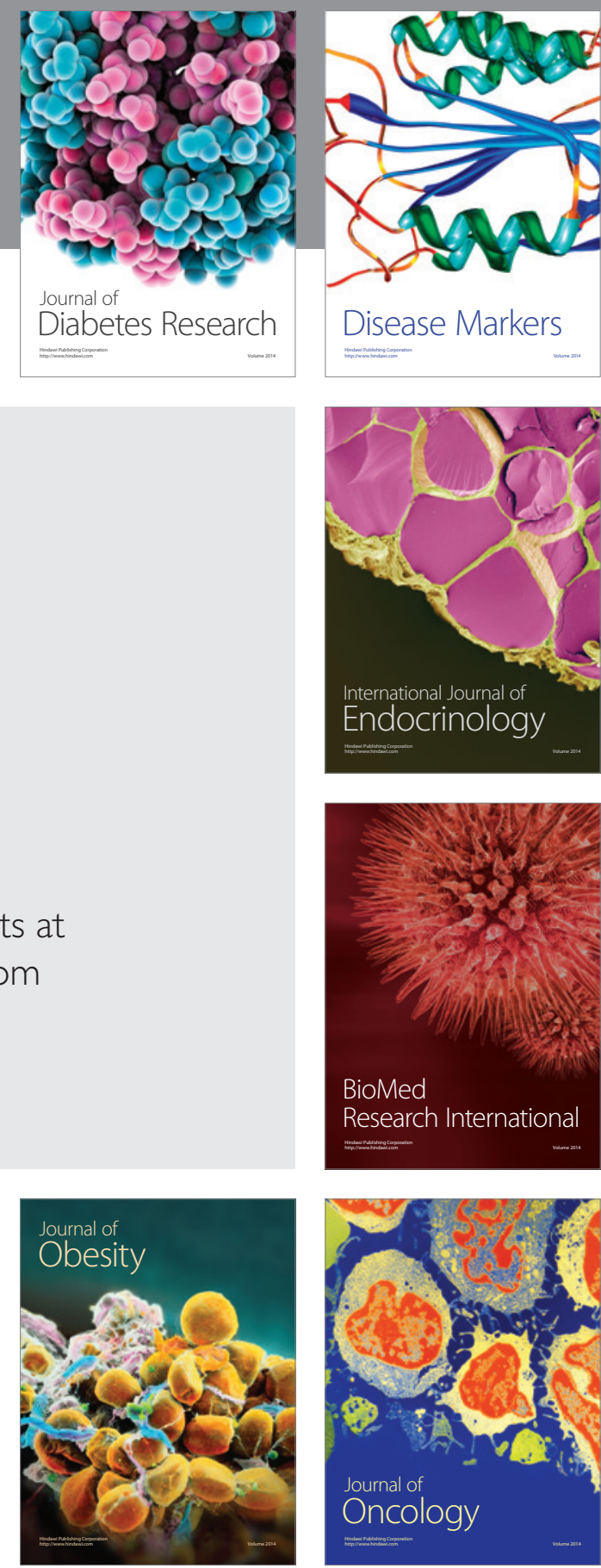

Disease Markers
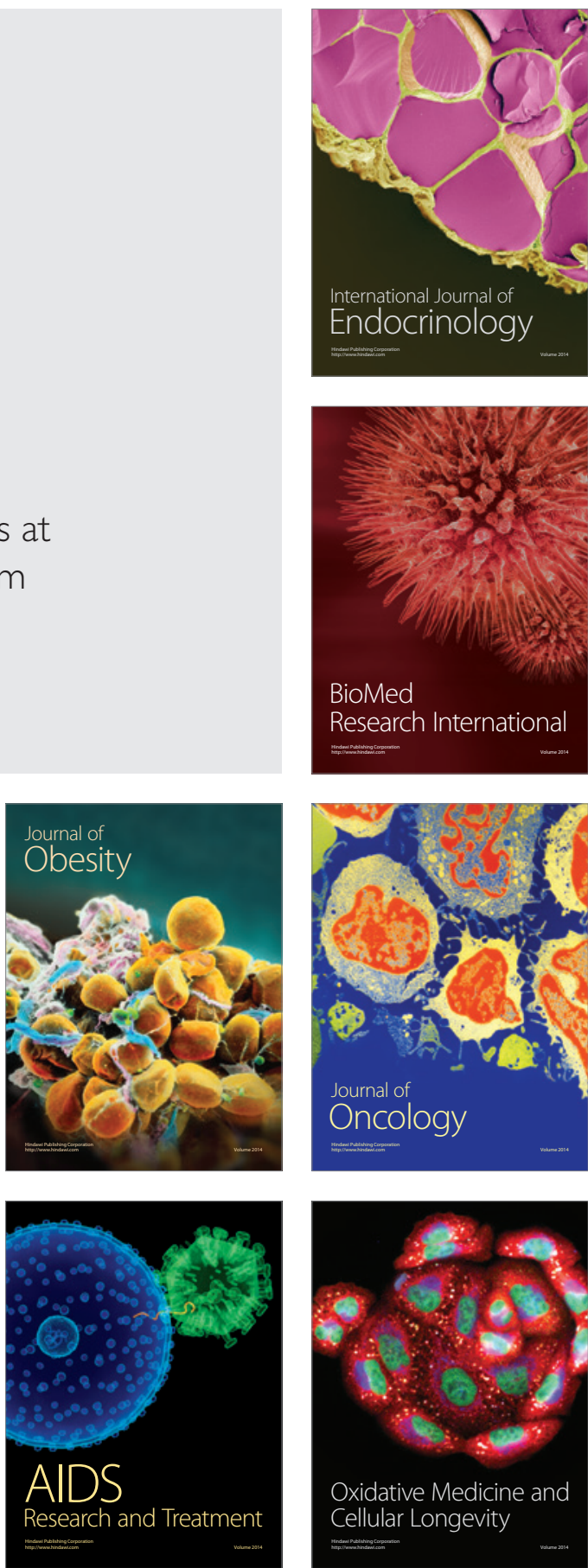